\title{
Uniform versus Nonuniform Axial Power Distribution in Rod Bundle CHF Experiments
}

\author{
Baowen Yang, Jianqiang Shan, Junli Gou, Hui Zhang, Aiguo Liu, and Hu Mao \\ Science and Technology Center for Advanced Fuel Research \& Development, School of Nuclear Science and Technology, \\ Xian Jiaotong University, Xian 710049, China
}

Correspondence should be addressed to Junli Gou; junligou@mail.xjtu.edu.cn

Received 30 December 2013; Accepted 7 May 2014; Published 27 May 2014

Academic Editor: Bin Zhang

Copyright (C) 2014 Baowen Yang et al. This is an open access article distributed under the Creative Commons Attribution License, which permits unrestricted use, distribution, and reproduction in any medium, provided the original work is properly cited.

Rod bundle experiments with axially uniform and nonuniform heat fluxes are examined to explore the potential limitations of using uniform rod bundle CHF data for CHF correlation development of light water reactors with nonuniform axial power distribution (APD). The case of upstream burnout is presented as an example of unique phenomena associated with nonuniform rod bundle CHF experiments. It is a result from combined effect of axial nonuniform power shape and different interchannel mixing mechanisms. In addition, several key parameters are investigated with respect to their potential impacts on the thermalhydraulic behaviors between rod bundles with uniform and nonuniform APDs. This type of misrepresentation cannot be amended or compensated through the use of correction factors due to the lack of critical information in the uniform rod bundle CHF testing as well as the fundamental difference in the underlining driving mechanisms. Other potential issues involved with the use of uniform rod bundle CHF data for nonuniform APD system applications also present strong evidence concerning the limitations and inadequacy of using uniform rod bundle CHF data for the correlation, prediction, and design limit calculation for safety analysis.

\section{Introduction}

In the light water reactors, one of the limiting conditions for safety operations is determined by the heat transfer capacity between the cooling water and the fuel clad, limited by the departure from nucleate boiling (DNB) or dryout (DO) phenomena, while local heat flux exceeds critical heat flux (CHF). Due to complex subchannel geometry as well as the broad range of thermal-hydraulic conditions, the measurement, prediction, and enhancement of the CHF limits for the light water reactor continue to be a challenging task [1]. The advancement of mixing vane grid and fuel assembly design increases the concerns of high flux density requirements that come with increasing fuel burnup and the broad range thermal-hydraulic conditions (extending from high pressure, high flow, down to low pressure, and low flow after the Fukushima accident, especially for the advanced reactor design using passive cooling concept) [2]. Furthermore, the expected CHF performance as well as power margin improvement is becoming a critical issue which not only demands design improvement for a high CHF performance mixing vane grid design, but also requires accurate and representative CHF measurement as well as predicting scheme. Since the closing of CHF test facility at Columbia University (CU-HTRF) [3], there is a growing concern not only for the lack of standard for the requirement on how and when the rod bundle CHF data from the newly established vendor-operated rod bundle CHF test facility should be independently verified or calibrated, but also on the trend of continuing relaxing requirements for $\mathrm{rod}$ bundle CHF correlation development and CHF prediction mechanism, as well as safety analysis evaluation.

In the past, the practice of conservative approach in analyzing, correlating, and applying any experimental data for nuclear design, operation, and safety analysis has limited any extrapolation of applicable ranges (operating or geometry range) supported by reliable and validated experimental data, especially when different thermal-hydraulic mechanism might be involved in the extended areas. Recently, with pressure from increasing demand on high power density 
and broader range of operating condition as well as the lack of fully benchmarked rod bundle CHF testing facility, progressive computational and statistical schemes are aggressively applied to analyze limited amount of available data through various optimal correlation and statistical analysis schemes. In some cases, the so-called "statistical poolability" scheme was applied to utilize unrelated CHF data (with different test geometries or conditions) and to extrapolate the application range of the CHF correlation to a totally untested domain for the particular fuel design disregarding significant difference in underlining fuel design characteristics as well as major difference in basic physical phenomena. Without actual improvement in the fuel design, the recent advancement in data treatment methodologies, the increasingly aggressive data analysis and optimization schemes, and the relaxing in requirements on rod bundle CHF experimental data have taken away more and more safety margins without proper experimental verification and supports. Similar voice was raised by Herer that "optimized correlations and advanced methodologies reduce this margin" [4]. This concern is especially valid considering the void of a totally benchmarked and fully established facility after the closing of Columbia University, Heat Transfer Research Facility (HTRF). Even with the continuous claims of so called "benchmarked facility" in recent publications $[5,6]$, most of the data presented in these papers were limited to very narrow range of test conditions with selected grid design only.

With the recent reducing safety margin in data analysis due to the overly optimized correlation and aggressive data treatment methodologies, the requirements for experimental accuracy as well as the reliability of "representation" between the simulated experiment and the targeted thermalhydraulic event have again become the focus of attention. In particular, several critical issues concerning the "accuracy" and/or "representation" in various aspects of rod bundle CHF experiments have become subjects of review and/or concern. These issues include, but are not limited to, the impacts of rod bow on CHF performance (Can the bow rod penalty derived from over 30-year-old uniform rod bundle CHF data with very primitive vaneless grids still be applicable for the current advanced fuel application?), the interpretation and usage of cold rod CHF data, the steadiness of rod bundle CHF testing operation, the effect of heater length, the impact of various experimental measurement uncertainties, and the application of uniform rod bundle CHF data in CHF correlation development, prediction, and safety analysis for the reactor core with nonuniform APD. Some examples of measurement uncertainties include the root cause and propagation of the measurement uncertainty of each individual measurement (especially the primary measurement, such as the inlet temperature, the flow rate, the heat flux measurement, etc.), the operating uncertainty (uncertainty and repeatability between experimental operations, between test bundles, and between test facilities), heat loss measurement and calculation as well as its application. Other potential concerned issues also include variations in experimental or operational design and setups, involving but are not limited to bundle tilt or misalignment, rod bow, excessive heat loss, bypass flow (in or out), uneven rod-to wall gap distribution, local flow blockage, and so forth.

In this paper, the particular issue of applying uniform rod bundle CHF data for the development of CHF correlation, prediction, and design limit safety analysis of nuclear reactor with nonuniform axial power distribution is examined. Several potential limitations and inadequacy in this type of application are explored. The limitations or lack of true representation in uniform rod bundle CHF data is mainly due to underlining deviations in simulating the thermal hydraulic phenomena leading to CHF event, which include but are not limited to the following.

(1) There is a lack of heated length effect or true heated length effect in uniform axial heated rod bundle CHF experiments. This type of heated length effect is normally observed in the CHF data from rod bundles with nonuniform axial power distribution under high quality, low pressure conditions.

(2) In the uniform rod bundle CHF test, there is also a lack of combined local heat flux and quality effect on initiating or triggering the critical heat flux limit that is dominated by either departure from nuclear boiling (DNB) or dryout (DO) events. With the uniform axial power distribution, the CHF (or physical burnout, including DO and DNB events) always occurs near the end of the heater length where the maximum quality is the predominating cause of driving the CHF event. In an axial uniform heated system, it is very difficult to simulate high subcooled DNB events with high subcooled, high local heat flux and relatively low local quality conditions as often observed in high axial heat flux peaking nonuniform heating systems.

(3) In the uniform rod bundle CHF testing, there is also lack of information related to burnout location driven by local heat flux and/or local heat flux gradient which can be only observed in a nonuniform APD heating system. Therefore, information from uniform rod bundle CHF data cannot be used to predict burnout location in a nonuniform rod bundle assembly. This is one of the main reasons why the burnout location predictability is usually very low for CHF correlation developed based on uniform CHF data or nonuniform CHF data with nonprototypical test conditions, heater length, or power profile. A recent example of PWR rod bundle CHF correlation developed based on mostly uniform data (with few 12 feet nonuniform rod bundle CHF data) demonstrates an extreme low predictability (below 50\%) of burnout location for 14 feet cosine nonuniform CHF test cases. For the same reason, in an exit peak skew power profile (top peak mu-sign-mu power shape) case, since most of the CHF events occur near the exit peak location, this type of nonuniform APD does not really contain much of variation in combining heat flux and quality impact on burnout location. Therefore, this type of data should not be used in the statistics in evaluating the location predictability of a correlation in predicting the CHF location for a system with axial nonuniform power profile.

(4) It is impossible to properly derive the needed $F$-factor using uniform rod bundle CHF test data. In fact, it would require several sets of nonuniform CHF data with different APDs, including axial uniform APD, to correlate and obtain 
a reasonable $F$-factor value to cover the conventional range of PWR thermal-hydraulic conditions.

(5) The effect of different grid spacing related terms (dg: distance to upstream grid; gsp: grid spacing; or Cgsp: current grid spacing, etc.) is very difficult or extremely complicated to simulate using heater rods with uniform axial power profile. This is especially true when intermediate flow mixers are used that lead to not only potentially different gsp or $\mathrm{dg}$ term with possibility of upstream burnout, but also the possibility of the effect from different upstream grid designs (either MVG or intermediate flow mixing device) and alignment.

(6) There is no information related to burnout location in a nonuniform APD heating system (except for the exit peak skew power distribution) that can be obtained in uniform APD rod bundle CHF experiments. In the past, uniform rod bundle CHF data obtained using different upstream grids (either regular MVG or intermediate flow mixing device (or flow mixer)) were treated indiscriminately. That is, the intermediate flow mixing device (MSMG, IFM, or any short form of the MVG that is used in between two regular MVGs) was treated as a regular MVG grid with uniform rod bundle $\mathrm{CHF}$. The only difference in this consideration is full span or half span. With gradually diverting design in not only mixing vane but also dimple, spring type, grid strap, and other key components, there is increasing potential of significant difference in thermal-hydraulic performance between regular mixing vane grid and the intermediate flow mixing grid/device. In a case example of intermediate flow mixing device (MSMG or IFM) application, different grid performance between regular MVG and the intermediate flow mixer was observed during the nonuniform heat flux CHF testing where the majority of CHF occurred at immediate downstream of regular MVG grid, instead of immediate downstream of the most expected intermediate flow mixer due to its relatively shorter grid height. This type of different mixing performance was very difficult to examine with uniform APD rod bundle CHF experiments. It was often mistakenly neglected when uniform heater rod was used for rod bundle CHF testing with only one grid spacing term where the particular grid type (MVG or intermediate flow mixer (or mixing device)) of the grid upstream of $\mathrm{CHF}$ event was not taken into consideration.

\section{Uniform ADP for Nonuniform ADP Application-Tong's F-Factor Approach}

In the history of rod bundle CHF correlation development, both uniform and nonuniform axial flux shapes (AFS) have been tested for various fuel designs.

A rod bundle CHF experiment with an axial uniform power distribution costs much less (at least two- to threefolds) than a similar rod bundle CHF testing using a nonuniform axial power distribution. With most relatively slow events (mostly dry out (DO) type CHF) and easily predicted burnout location (near the end of heater rod), the rod bundle $\mathrm{CHF}$ experiment with uniform axial heat flux distribution is a much easier option than a nonuniform APD. Based on both economic and technical considerations, uniform APD is often used as the substitute for the much challenging nonuniform APD rod bundle CHF experiments for the purpose of $\mathrm{CHF}$ correlation development, $\mathrm{CHF}$ prediction, and design limit (DL) safety analysis.

In order to apply CHF data from uniform APD rod bundles for Non-uniform APD reactor core application, it is a common practice to apply Tong's $F$-factor for power shape correction to account for the total accumulated energy input at any given axial elevation.

It is well known that, for predicting $\mathrm{CHF}$, a method has been derived by Tong through introducing an $F$-factor to account for the effect of the heat flux profile on the local $\mathrm{CHF}$ value. Derived from empirical correlation based on data obtained using rod bundles with different APDs, the $F$-factor is often used for test bundles with both uniform and nonuniform power profiles to account for the energy accumulated in the bubble layer up to the concerned location [7]. Most PWR CHF correlations, like W-3 and FC2000, use this method to consider the impact of the axial heat flux shape. The $F$-factor is defined as

$$
F=\frac{q_{\mathrm{CHF}, U}}{q_{\mathrm{CHF}}}
$$

where $q_{\mathrm{CHF}, U}$ is the CHF calculated by means of the correlation for a axially uniform heat flux shape and $q_{\mathrm{CHF}}$ is the $\mathrm{CHF}$ in the case of a nonuniform heat flux. An energy balance for the bubble layer provides the analytical expression of $F$ (Tong):

$$
F=\frac{K}{q\left(l_{\mathrm{DNB}}\right)\left(1-e^{-K\left(l_{\mathrm{DNB}}-l_{o}\right)}\right)} \int_{l_{o}}^{l_{\mathrm{DNB}}} q(z) e^{-K\left(l_{\mathrm{DNB}}-z\right)} d z,
$$

where $l_{\mathrm{DNB}}$ is the location of DNB, $l_{o}$ is the elevation of onset of the boiling, $q(z)$ is the local heat flux and $K$ is an experimental coefficient describing the heat and mass transfer effectiveness at the bubble-layer/subcooled-liquidcore interface as given by

$$
K=\frac{b_{1}(1-X)^{b_{2}}}{G^{b_{3}}},
$$

$X$ is the local quality, $G$ is the local mass flux, and $b_{1}, b_{2}$, and $b_{3}$ are optimized coefficients of the $F$. These coefficients are usually optimized using the non-uniform rod bundle $\mathrm{CHF}$ data. These values are usually different depending on each correlation. For example, in BW-2 Correlation, $b_{1}=0.15, b_{2}=$ 4.31 , and $b_{3}=0.478$; on the other hand, in W-3 Correlation, $b_{1}=0.44, b_{2}=7.9$, and $b_{3}=1.72$, and so forth.

The $F$-factor indicates the memory effect of the upstream heat flux profile on the $\mathrm{CHF}$ and accounts for the difference in the amount of energy accumulated in the bubble layer up to the location of interest. The quantity $1 / K$, which shows up in the exponential function, defines an exponentially decaying behavior with respect to the axial distance below the $\mathrm{CHF}$ location because $K$ decreases as the quality increases as given in (3). The starting elevation of integration may be taken to be either the inception of local boiling or the inlet. In the limit of very high qualities $(X>0.2)$, DNB is no longer 
the dominant CHF mechanism, and the occurrence of CHF might be considered as a global phenomenon, with little dependence on the local value of the heat flux.

\section{Challenges to the Application of Uniform APD CHF Experiment to Simulate a Non-Uniform APD System}

The above $F$-factor is based on integrated energy approach considering only total energy input up to a certain local elevation for a uniform or nonuniform heated channel. This $F$-factor correction reflects mostly the total quality up to the concerned elevation, without any implication or gradient of the local heat flux. The application of $F$-factor heavily relies on the assumption that the DNB is no longer a dominant CHF mechanism.

Although adjustment factor, such as Tong's $F$-factor, is usually applied for correction of uniform CHF data for Nonuniform APD, this local adjustment does not represent the true local condition, especially the condition that might have impacts on the mixing performance as well as the occurring of DNB, such as local heat flux peaking.

As shown in Figure 1, for the axially uniformly heated test section, the CHF will most likely occur at the exit of the test section where the quality is at its maximum. That is, the CHF will most likely occur as a maximum quality driven event. On the other hand, for an axially nonuniform heated rod, with the exception of exit peak skew power shape, the CHF does not always occur at a certain elevation (exit peak or channel exit); rather, it often occurs at a location based on the combined effect of local quality and heat flux peaking. For an axial cosine power shape, the actual burnout (or CHF) often occurs at various elevations, from the midspan area (upstream and downstream of mid-span) all the way up to near about $10 \%$ from the end of heated length (but not at the end of heated length, except for a very extremely low peaking power shape such as the uniform axial APD case or exit power peaking case). The actual burnout location for an axially nonuniform power distribution heater tube is depending on the operating condition, the actual power shape, grid alignment/configuration, the grid span, and, most importantly, the actual mixing and turbulent promoting performance of the mixing vane grid.

In a typical nonuniform APD case of rod bundle CHF with a cosine axial power distribution, under normal operating condition and similar mixing vane grid and similar grid spacing at each elevation, as shown in Figures 2 and 3, the CHF often occurs near $2 / 3$ elevation of the entire heated length. However, the actual mixing effect of any given mixing promoter at any elevation is often affected by the type of mixing promoters, difference in grid spacing, mixing vane grid alignment, and, most importantly, the difference in local thermal-hydraulic conditions, including not only the local quality, local liquid, and vapor superficial velocity, but also the local heat flux as well as the gradient of local heat flux itself. Depending on the type of grid design, these local conditions might have significant impacts on mixing vane performance in terms of cross flow mixing effect (cross flow factor), local

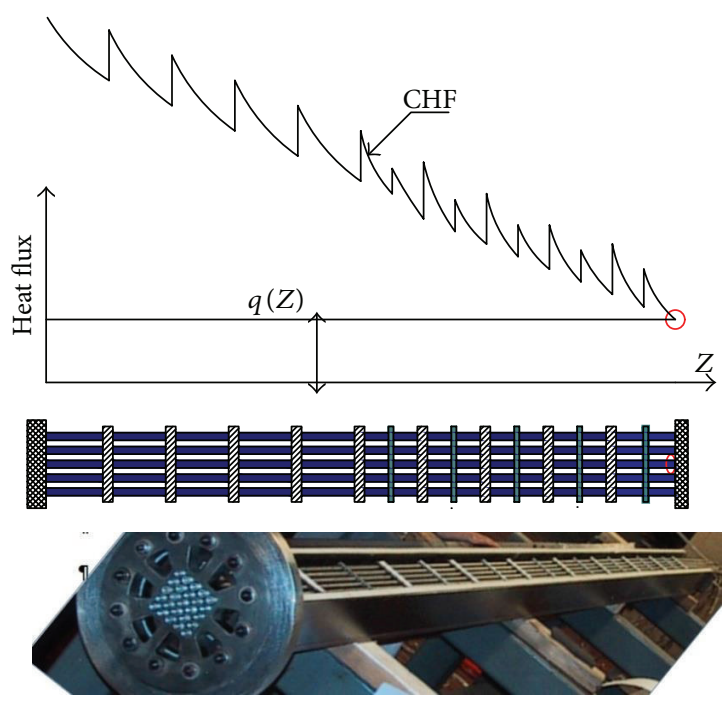

FIGURE 1: Rod bundle CHF with uniform axial power distribution. (CHF mostly occurs at the end of heated length.)

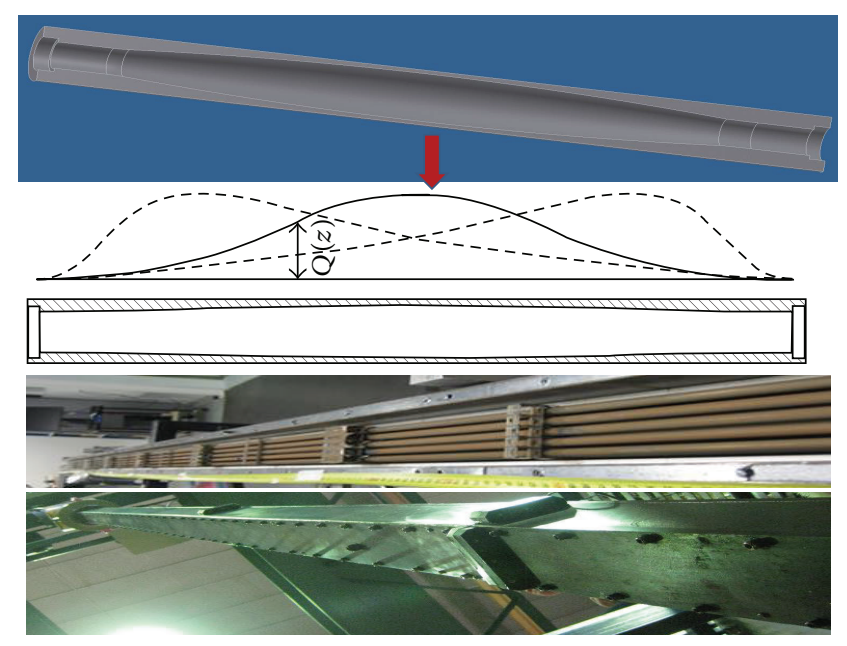

FIGURE 2: Rod bundle CHF with nonuniform axial power distribution.

turbulent intensity, angular moment, and presence of swirl flow (swirl ratio factor or swirl mixing ratio), as well as twophase flow bubble and turbulent dynamics. The actual mixing effect will in turn not only impact the actual CHF power level and influence the CHF mechanism, but also affect the elevation and location where burnout (or CHF) occurs. In summary, the performance of mixing promoters is dependent on both the design of the mixing grids and the local thermalhydraulic conditions. For example, as shown in Figure 4, when two different grids are applied in the same bundle assembly (in the most common cases of regular mixing vane grid (MVG) with intermediate flow mixing device) since the regular MVG and the intermediate flow mixing device do not always have exactly the same grid design (even though they might have the same vane shape, size, and pattern, the spring, dimple, and the height are often different from each other), 


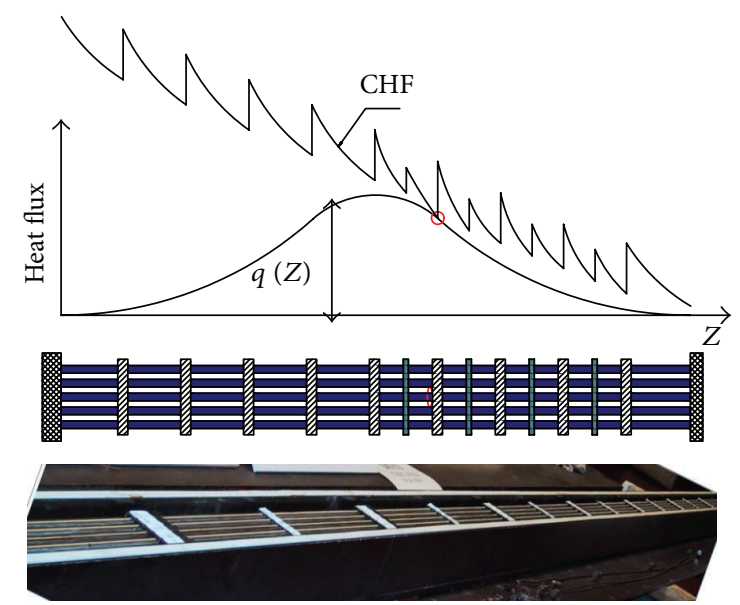

FIgURE 3: Rod bundle CHF with nonuniform APD. With the example of cosine axial power distribution, under normal operating condition and similar mixing vane grid and similar grid span at each elevation, the CHF mostly occurs near $2 / 3$ elevation of the entire heated length.

the CHF burnout location can vary depending on the actual grid location, the grid spacing, the gird alignment, and local operating conditions (see Figure 5). The difference in mixing performance between regular MVG and the intermediate flow mixing device is often overlooked in the CHF tests using rod bundle with uniform APD.

As a matter of fact, with two or more different mixing vane grid designs (such as regular MVG and the intermediate flow mixing device) in the same test bundle, the location/elevation of CHF occurrence in the nonuniform APD bundle experiment could be a good indication of which type of grid has better CHF performance. For example, as mentioned above, if the MVG and intermediate flow mixing device are alternatively positioned along the heater length, based on conventional wisdom, most of CHF events would occur directly downstream of intermediate flow mixing device due to the fact that it is a relatively shorter grid with lower pressure drop. Therefore, the regular MVG should have better mixing performance than the much shorter and simpler intermediate flow mixing device. However, in the past, the opposite situation was also observed (CHF occurred directly downstream of regular MVG grid) for a certain type of intermediate flow mixing devices. This type of $\mathrm{CHF}$ events provide strong indication that intermediate flow mixing device, although shorter, might produce better mixing performance than the taller version of regular mixing vane grid. This type of CHF data from non-uniform rod bundle CHF experiments provide clear evidence that MVG and intermediate flow mixing devices are indeed different from each other, with different thermal-hydraulic performance, and should be considered as two separate grid types. They should have different mixing performances with different TDCs (thermal diffusion coefficient) and should be handled differently in the CHF correlation, prediction, and safety analysis processes. In the case of CHF testing with uniform APD, the CHF will most likely occur at the end of heated

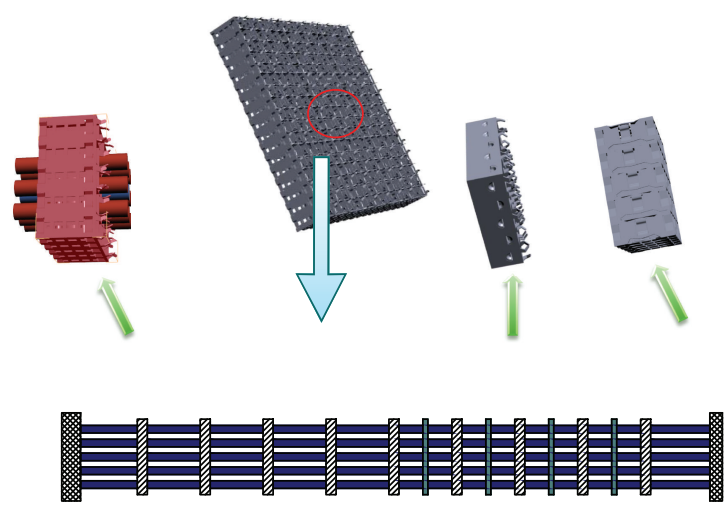

FIGURE 4: A rod bundle assembly with grids of different designs located at various elevations with different grid spans and different grid alignments.

length independent of the upstream alignment of mixing vane grid, either regular MVG or intermediate flow mixing device.

In the following illustration case of the rod bundle CHF experiment, the nonuniform CHF experiment has most (or all) of the CHF thermocouple indications located at level 4 (T/C\#4), downstream of regular MVG (Mixing Vane Grid) instead of level 3 or level 5. This is an indication that, for these particular grids, although the intermediate flow mixing device (MSG or IFM, etc.) is a much shorter grid with lower pressure drop and similar vane pattern, it has better $\mathrm{CHF}$ performance than regular MVG.

In the above case, if a bundle assembly consists of two or more types of mixing vane grids, such as regular MVG and intermediate flow mixing device (mixer), and so forth, the alignment of the last two grids (assuming the grid directly upstream of the burnout location has the most direct impact on the CHF performance) becomes a major challenging decision for a CHF test using a rod bundle with uniform axial power distribution. In this case, the conventional arrangement of having intermediate flow mixing device (MSMG or IFM) at the middle of the last span of the bundle could be a nonconservative approach, because it will be mostly measuring the $\mathrm{CHF}$ performance of a higher performing grid (MSMG or IFM) rather than the lower CHF performance of a MVG grid. Detailed discussion on this can be seen in the following section.

As shown in Figure 6, considering the potential difference in thermal-hydraulic performance between the regular MVG and the intermediate flow mixing device, the last two grid alignments can be various in many ways, not only the grid spacing, but also the alignment between the regular MVG and the intermediate flow mixing device (IFM, MSMG, etc.) in order to truly represent or simulate the CHF events in a nonuniform axial power distribution (APD) system, such as the reactor core. In a most common cosine power shape case, with the most popular alignment of having intermediate flow mixing devices only used at the downstream half of the fuel assembly, since the CHF could happen upstream or 


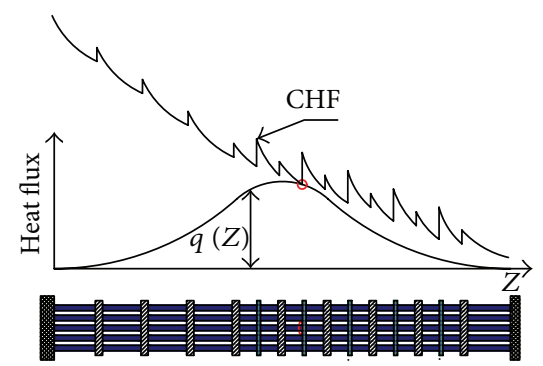

(a)

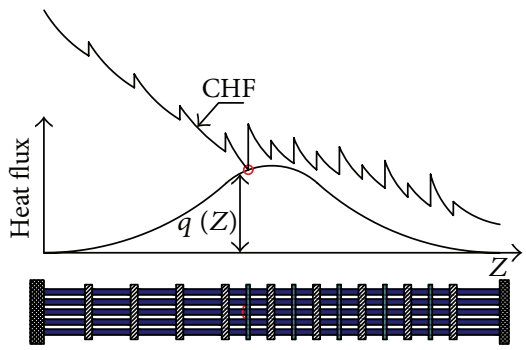

(b)

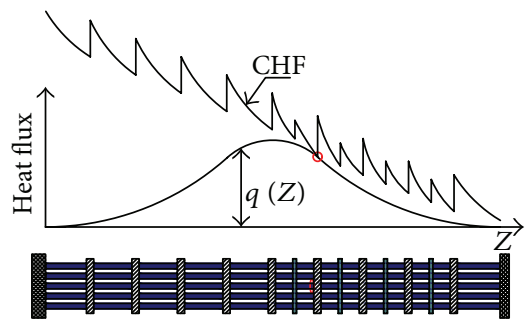

(c)

FIGURE 5: Actual burnout (CHF) location in a rod bundle with cosine APD can vary depending on the actual grid location, the grid span, and the gird alignment, as well as local operating conditions.

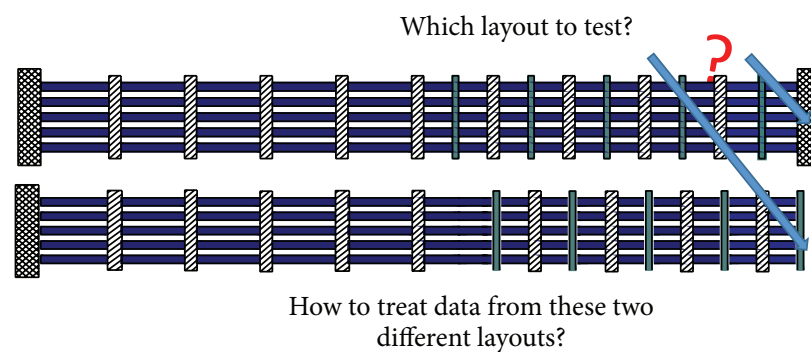

(a) Two examples of potential uniform bundle

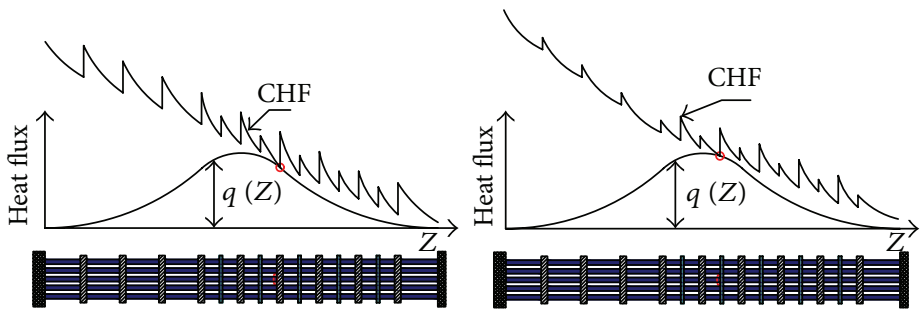

(b) Two potential CHF events in a nonalignments uniform APD bundle

Figure 6: In uniform APD CHF experiment, the last two grid alignments can be various in many ways (only two examples are illustrated here (see Figure 6(a)) in order to simulate the occurring of CHF events in a nonuniform APD system (see Figure 6(b))).

downstream of the mid-span of the rod bundle, the last gird span could be a full span or a half span alignment. In order to account for the impact from the most affective grid, which is just at the upstream of the burnout location, various mixing vane grids, grid configurations, and grid spacing (half or full grid span) must be examined. Furthermore, considering the potential impact of (incoming) local thermal-hydraulic conditions on the mixing performance of a mixing vane grid at any given elevation, it is also important to take into account the accumulated mixing performance from the upstream mixing vane grids and their alignment. That is, for any rod bundle CHF testing with different grid spacing and involving different grid types (both regular MVG and intermediate flow mixing devices), if rod bundle CHF tests are performed using uniform axial power distribution bundle assembly to simulate $\mathrm{CHF}$ or thermal hydraulic event in a nonuniform axial power distribution system, all the potential combinations of grid type, grid spacing (full span and half span or any other span actually used in the reactor), and grid alignments should be performed in order to include all possible events covering the basic underlining physical phenomena. It will be also a major challenge to handle, interpret, and analyze all the data obtained from the above listed potential combined configurations with proper Tong's $F$-factor (or $F$-factors) or other correction factor in order to apply this type of uniform rod bundle CHF data for nonuniform power shape system application.

3.1. Summary of Challenges and Limitations in Applying Uniform APD Rod Bundle CHF Data for Nonuniform APD Rod Bundle Applications. As described above, some potential constraints and challenging issues involved in the use of $\mathrm{CHF}$ data from uniform rod bundle CHF testing for nonuniform axial power shape heating application are summarized as follows.

(1) It is extremely difficult or impossible to use a rod bundle with uniform APD to simulate the CHF event that occurs 
in a nonuniform APD system. This dissimilarity presents the fundamental difference or deficiency in simulating a nonuniform rod bundle $\mathrm{CHF}$ using uniform rod bundle CHF data.

(2) Effect of heated length it is well known that as long as the total length of the heater rod is longer than the mixing length of the mixing grid, CHF testing with uniform APD will most likely reveal no heater length effect on measuring CHF. On the other hand, effect of heated length on CHF is often observed in case of nonuniform APD, especially under nonlocal CHF event.

(3) There is a lack of prototypical combined local heat flux and local quality driven mechanism leading to the CHF limit or to the incipient of heat transfer deterioration. That is, in case of uniform APD, CHF always occurs at $X_{\max }$. It is a maximum quality driven and dominating event with very limited local heat flux effect. With the axial uniform heat flux profile, it is rather difficult to simulate high subcooled DNB behavior. This is quite different from a nonuniform APD case, where CHF or DNB seldom or never occurs at the point of maximum quality. Just like axial nonuniform power profile in the reactor core, the CHF limit in a nonuniform axial heat flux experiment often reaches a combined effect of local quality and local heat flux (sometimes even local heat flux gradient). In a channel with axial non-uniform power input, the potential CHF mechanism covers wide range of thermal-hydraulic phenomena from high quality dryout, to relatively subcooled DNB event, to potentially homogeneous nucleation behavior (to be discussed in a separate paper) a mainly local heat flux driven event which might occur off the heater wall at or before the NVG (Net Vapor Generation) point. In the non-uniform case, since the CHF never occurs at the end of heater rods, in some cases, integral effect has major impact on the thermal hydraulic behavior, especially in the low flow, low pressure flow instability case. This type of integral effect will not happen in the uniform heater rod cases where CHF mostly occurs with the maximum quality at the end of heater rod.

(4) In a uniform heated test section, it is very difficult or impossible to reach high subcooled/high flux and relatively low local quality condition DNB event as often seen in high axial peaking nonuniform power shape axial heating systems.

(5) It is rather impossible to derive the needed $F$-factor using uniform rod bundle CHF test data alone. In fact, it would require several sets of nonuniform $\mathrm{CHF}$ data with different APD including axial uniform APD to correlate and obtain a reliable $F$-factor value. Usually, for the three coefficients to be optimized in the Tong's $F$-factor, for any given geometry (one particular heater length, or grid span, or test configuration, etc.), at least three sets of nonuniform rod bundle CHF test data should be obtained. More sets of CHF data with different power shapes or test configurations should be required if more than one particular set of test geometries are needed for the CHF correlation to cover. That is, the $F$-factor requires several sets (usually 6 sets or more CHF tests are needed to cover a minimum of two configurationstypical cell and thimble cell configurations) of nonuniform CHF data with different APD including axial uniform APD, to correlate and obtain a correcting factor in order to cover various power shape or axial distribution profiles that might occur in the reactor throughout the life cycle of the particular fuel.

(6) No information related to burnout location in a nonuniform APD heating system can be obtained from uniform APD rod bundle CHF experiments.

In the nonuniform heat flux (APD) case, the location of CHF (DNB or DO) usually depends on the power shape (cosine, top peak, bottom peak, etc.), grid design and alignment, and heater length, as well as other global and local test conditions which are designed to simulate the actual thermal-hydraulic system. Therefore, it is critical for a CHF correlation derived from the rod bundle CHF data to include information relating the actual burnout location to its dependent variables. A CHF correlation developed based on sufficient sets of CHF test data (normally 6 sets or more) with at least four or more different power shapes is needed to achieve reasonable burnout location prediction accuracy.

On the other hand, since all or most of the CHF occur at the end of the heater rod, there is no burnout location information relating to local heat flux revealing in the CHF data using rod bundles with uniform heat flux (APD). Subsequently, the CHF correlation developed based on uniform rod bundle CHF data will not be able to predict actual burnout location for a nonuniform heat flux (APD) heating system. One good example of the issue involving burnout location predictability is one of the latest released PWR CHF correlations for 14 feet bundle application. This correlation was developed based on mostly uniform rod bundle CHF data plus some 12 feet cosine bundle data points. Unfortunately, without proper information (only one set of 14 feet cosine bundle CHF data), this correlation can only predict the burnout locations for the 14 feet cosine case with lower than $50 \%$ success rate. Although an additional set of exit peak skew bundle CHF data was added to raise the "so-called" nonuniform power shape burnout location predictability, this high prediction accuracy should not be credited for its general burnout location prediction accuracy because, in the exit peak case, most of the CHF events as expected should occur near the exit peak location. High prediction accuracy in the case of exit peak skew power shape has no guaranty or relevance to the prediction accuracy of other nonuniform APD cases.

Based on the above illustration, it is very clear that there is lack of information from uniform rod bundle CHF data related to burnout location in a nonuniform APD heating system. This deficiency presents a major difficulty for using uniform rod bundle CHF data to predict burnout location in a nonuniform rod bundle assembly. Unfortunately, this inability of predicting other burnout locations of the nonuniform rod bundle CHF testing results is often misleadingly reported by the vendor as a SPECIAL capability of their CHF code in predicting CHF location precisely and repeatedly for their test cases of another uniform rod bundle CHF tests (always at the end of heated length).

(7) With a uniform axial flux shape heater, it is impossible to obtain data with upstream burnout as observed in a nonuniform axial flux shape system with high performance mixing vane grids. 

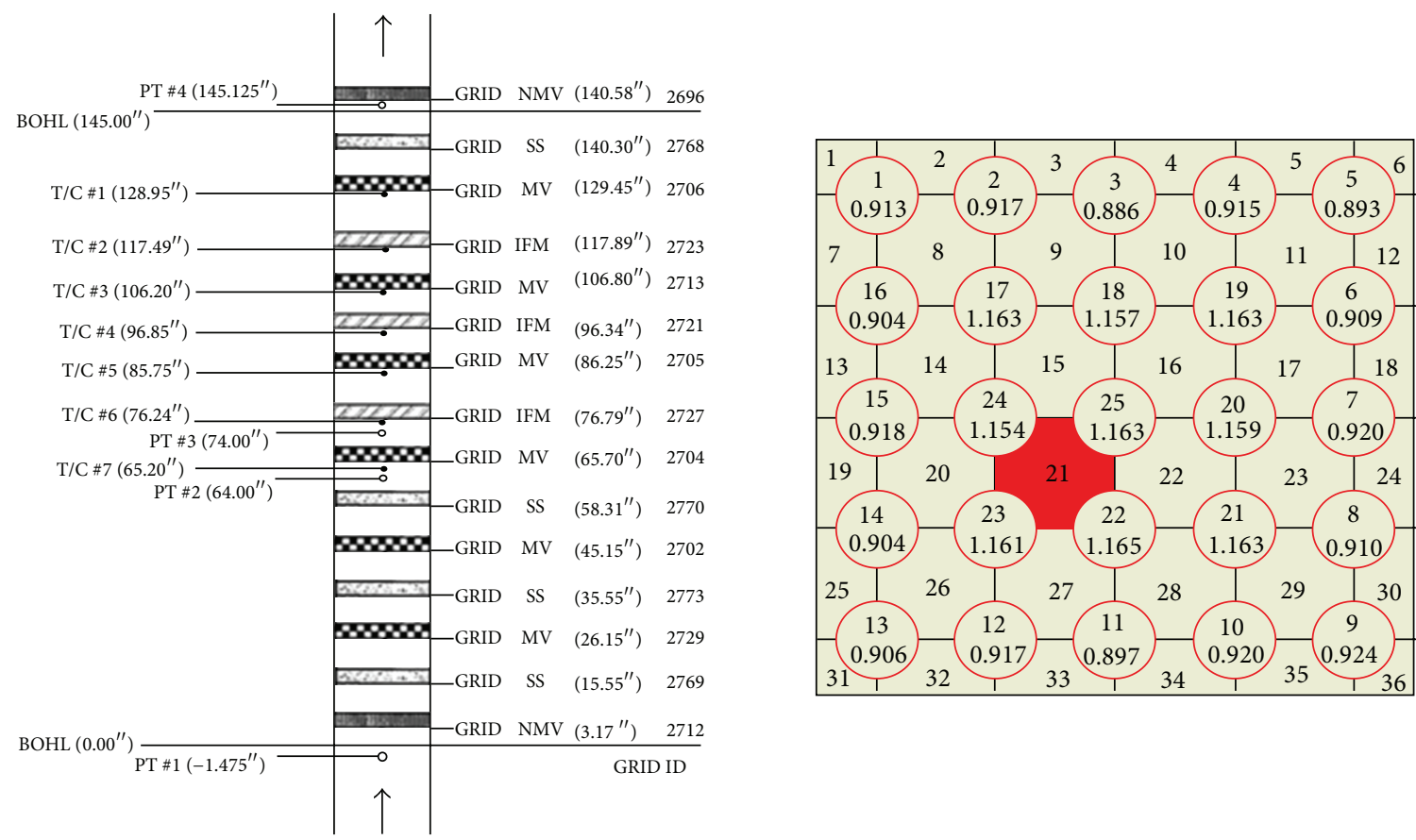

FIgURE 7: A case example of a $5 \times 5$ bundle configuration.

As shown in Figure 5, for more and more nowadays high performance mixing vane grids, there is an increasing frequency that burnout occurs upstream of a midspan, referred to as an upstream burnout. In a heated channel with axial cosine power profile, the upstream burnout usually occurs upstream before the midspan (50\% elevation), with the same local heat flux but lower local quality than the correspondent position downstream of the midspan. In this case, $\mathrm{CHF}$ occurs upstream of midspan most likely due to effective heterogeneous mixing downstream or possible homogenous nucleation mechanism (to be discussed further in a separate paper) at upstream location. This type of upstream burnout occurs more often for the fuel assembly with high mixing performance grids under relatively high flow rate, high local subcooled, and high local heat flux conditions. This type of upstream burnout phenomena will never occur in CHF experiments with uniform APD rod bundles. This is another example that $\mathrm{CHF}$ data obtained from uniform axial power distribution rod bundles cannot closely resemble thermal hydraulic condition in the reactor and should not be used for the safety analysis of nuclear power plant. The upstream burnout phenomena also demonstrate accumulated quality (or energy) type of correction factor, such as the F-factor, incapable of accurately reflecting such unique thermalhydraulic phenomena associated with local heat flux effect in an axial high peaking nonuniform heat flux (nonuniform APD).

One of the rod bundle CHF experiments with upstream burnout data is illustrated in the following case example. As shown in the test geometry (Figure 7) for a series of rod bundle $\mathrm{CHF}$ tests performed in a $5 \times 5$ test geometry, there are at least 7 upstream $\mathrm{CHF}$ data points (out of $52 \mathrm{CHF}$ points) occurring at thermocouples level number 7 (below midspan elevation), where, with similar local heat flux and axial cosine power distribution, the local quality is lower at level 7 as compared to its correspondent downstream location. One example is Run number 49 , with $G=3.52 \mathrm{Mg} / \mathrm{m}^{2} \mathrm{~s}, P_{\text {exit }}=$ $1800 \mathrm{psi}, T_{\text {inlet }}=576.8 \mathrm{~F}$, and CHF occurred at TC number 7 at rod number $21(\mathrm{~T} / \mathrm{C} 21.7)$ with $\mathrm{CHF}$ power of $2.31 \mathrm{MW} / \mathrm{m}^{2}$. Figure 8 gives the local equilibrium qualities and local mass fluxes in subchannels at different axial locations. This type of upstream burnout will not happen in an experiment with axially uniformly heated rod bundle.

(8) Effect of different grid spacing term (dg and gsp or Cgsp) is very difficult or complicated to derive from CHF data obtained from heater rods with uniform axial power profile.

This is especially true when intermediate flow mixers are used; they lead to not only potentially different gsp or dg term with potential upstream burnout but also possibly different upstream grid design (either MVG or intermediate flow mixing device/grid).

In the past, in case of fuel assembly with only one type of mixing vane grid (MVG) and one fixed grid span, the uniform rod bundle CHF test was conducted using only one type of simple configuration. However, with the frequent use of intermediate flow mixer and different grid spacing, the uniform rod bundle CHF simulation should take into account both vane type and grid spacing effect. The difference between these two types of mixing grids (MVG and intermediate flow mixing device) was not only verified by the CFD modeling, but also clearly indicated in the actual nonuniform rod bundle CHF experiments as discussed in the previous section. As shown in Figures 5 and 6 and the discussion above, in order to simulate different fuel 


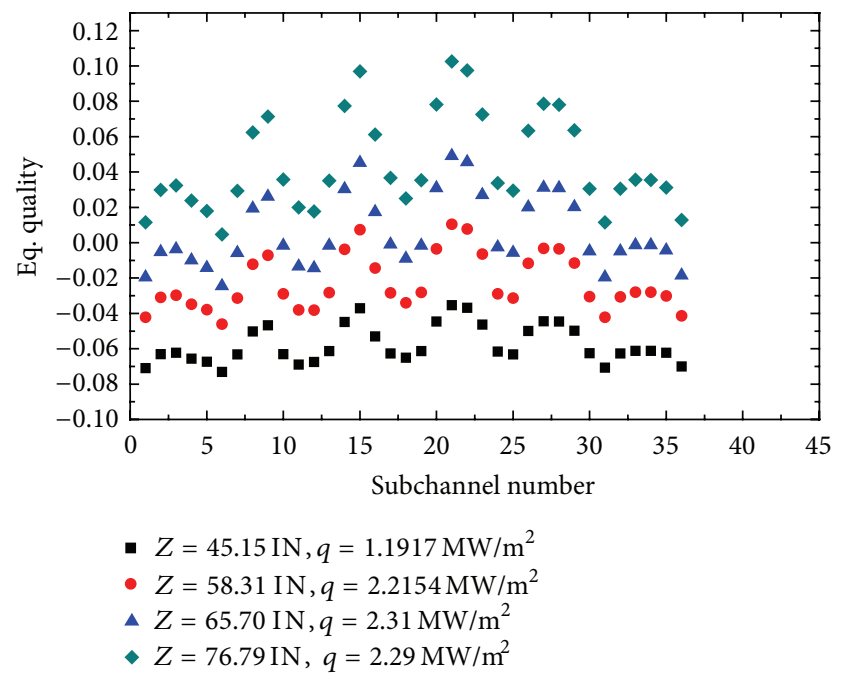

(a) Equilibrium quality

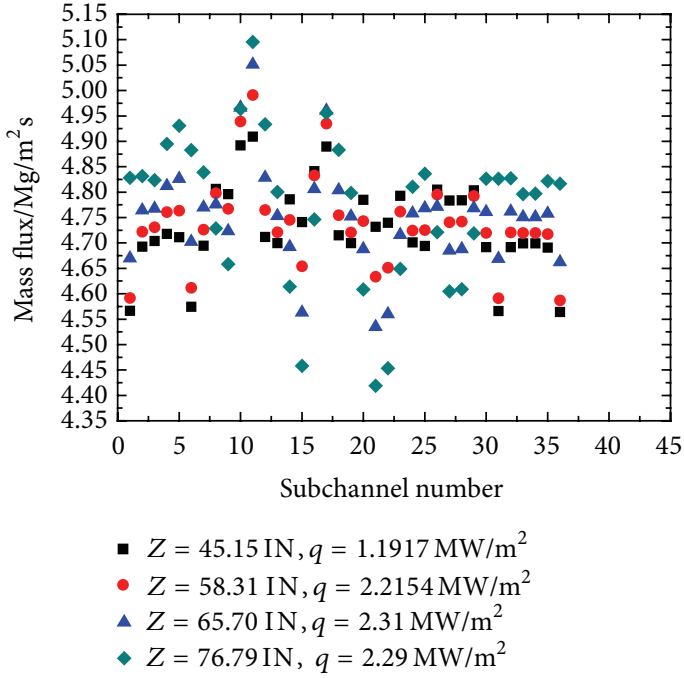

(b) Mass flux

FIGURE 8: Subchannel equilibrium quality and mass flux at different grid levels.

alignments for the last span and the effect of grid type for the grid upstream of the burnout location, there are many potential combinations of grid alignments, grid spacing, and overall power shape in order to simulate the local thermalhydraulic conditions leading to the true CHF events of interest. The interpretation and analysis of such uniform rod bundle CHF data as well as the prediction capability (both $\mathrm{CHF}$ power and $\mathrm{CHF}$ location) derived from this type of data should be a subject of major challenge.

\section{Conclusion and Recommendation}

Both uniform and nonuniform axial power distributions (APDs) have been tested for rod bundle CHF experiments. Although adjustment factor, such as Tong's $F$-factor, is usually developed through correlation and applied as a correction factor to account for the total accumulated energy input when compared to data obtained from nonuniform heater, this local adjustment does not represent the true local condition, especially the condition that might have an impact on the occurring of DNB, such as local heat flux as well as local heat flux gradient. Considering the above described limitation or deviation of using uniform rod bundle CHF data and the fact that this type of deviation cannot be amended or compensated through the use of correcting factor such as Tong's $F$-factor, it is obvious that the current use of uniform rod bundle $\mathrm{CHF}$ data for nonuniform power core safety analysis can be nonprototypical and nonconservative (especially the potential of missing extremely high subcooled DNB type data) and should be investigated or explored further. With nowadays advanced statistical analysis approach and aggressive utilization of $\mathrm{CHF}$ power margin, the use of uniform rod bundle $\mathrm{CHF}$ data for safety analysis of axially nonuniformly heated reactor core definitely calls for further critical reexamination.

\section{Conflict of Interests}

The authors declare that there is no conflict of interests regarding the publication of this paper.

\section{References}

[1] W. Liu and H. Nariai, "Viewpoint of subcooled flow boiling critical heat flux mechanism," Chemical Engineering \& Technology, vol. 25, no. 9, pp. 447-453, 2002.

[2] D. K. Chandraker, P. K. Vijayan, D. Saha et al., "A comprehensive review of the CHF prediction methodologies for the fuel rod bundle," in Proceedings of the 13th International Meeting on Nuclear Reactor Thermal Hydraulics (NURETH-13 '09), Paper: N13P1029, Kanazawa, Japan, September 2009.

[3] CU-HTRF-2001-W1010, Critical Heat Flux Tests on PWR Fuel Assemblies for Westinghouse Electric Company Test No. 101.0, 2001.

[4] C. Herer, "Questions relative to chf testing for new pressurized water reactor advanced fuel assemblies," in Proceedings of the 18th International Conference on Nuclear Engineering (ICONE18 '10), pp. 729-736, Xi'an, China, May 2010.

[5] L. D. Smith, A. Hallehn et al., "Benchmark testing the oden CHF loop to Columbia University HTRF," in Proceedings of the 14th International Meeting on Nuclear Reactor Thermal Hydraulics (NURETH-14 '11), NURETH-14-368, Ontario, Canada, September 2011.

[6] C. Herer, A. Beisiegel, P. Imbert et al., "Comparison of PWR fuel assembly CHF tests obtained at three different test facilities," in Proceedings of the 11th International Meeting on Nuclear Reactor Thermal Hydraulics (NURETH-11 '05), Paper: 117, Avignon, France, October 2005.

[7] L. S. Tong and Y. S. Tang, Boling Heat Transfer and Tow-Phase Flow, Taylor and Francis, Washington, DC, USA, 1997. 


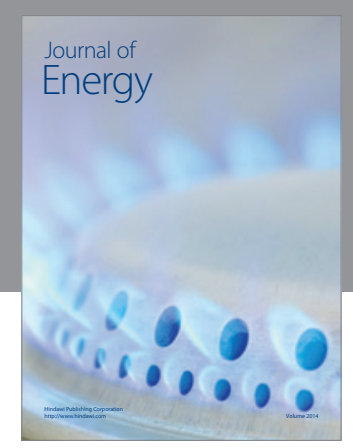

Journal of

Industrial Engineering
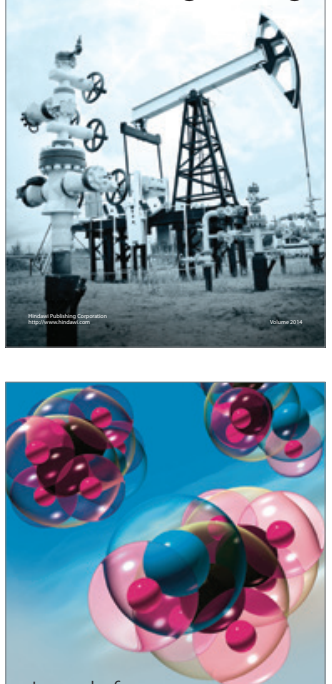

Fuels
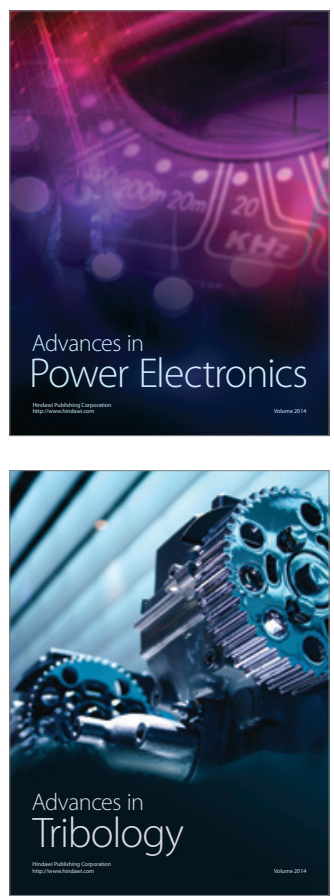

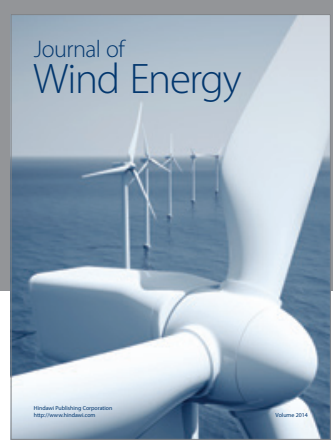

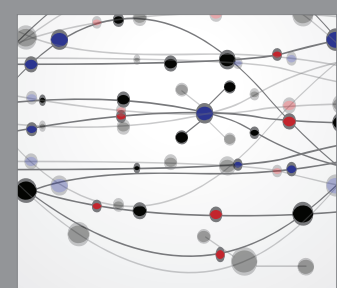

The Scientific World Journal

Submit your manuscripts at http://www.hindawi.com

Journal of

Structures
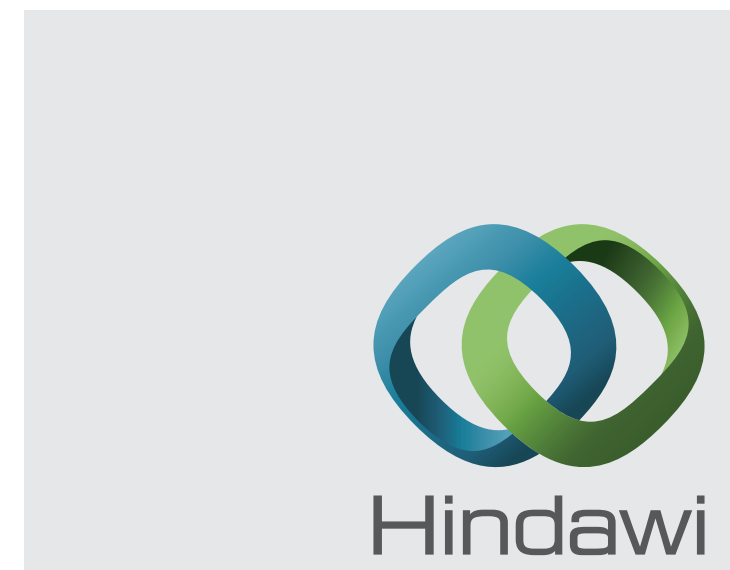

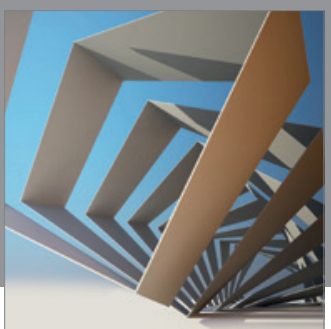

Rotating

Machinery
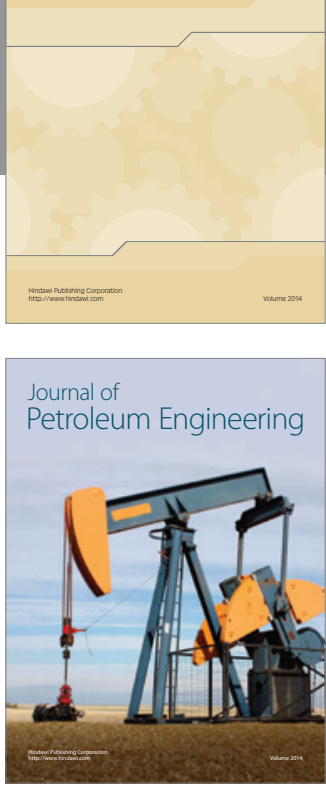

Journal of

Solar Energy
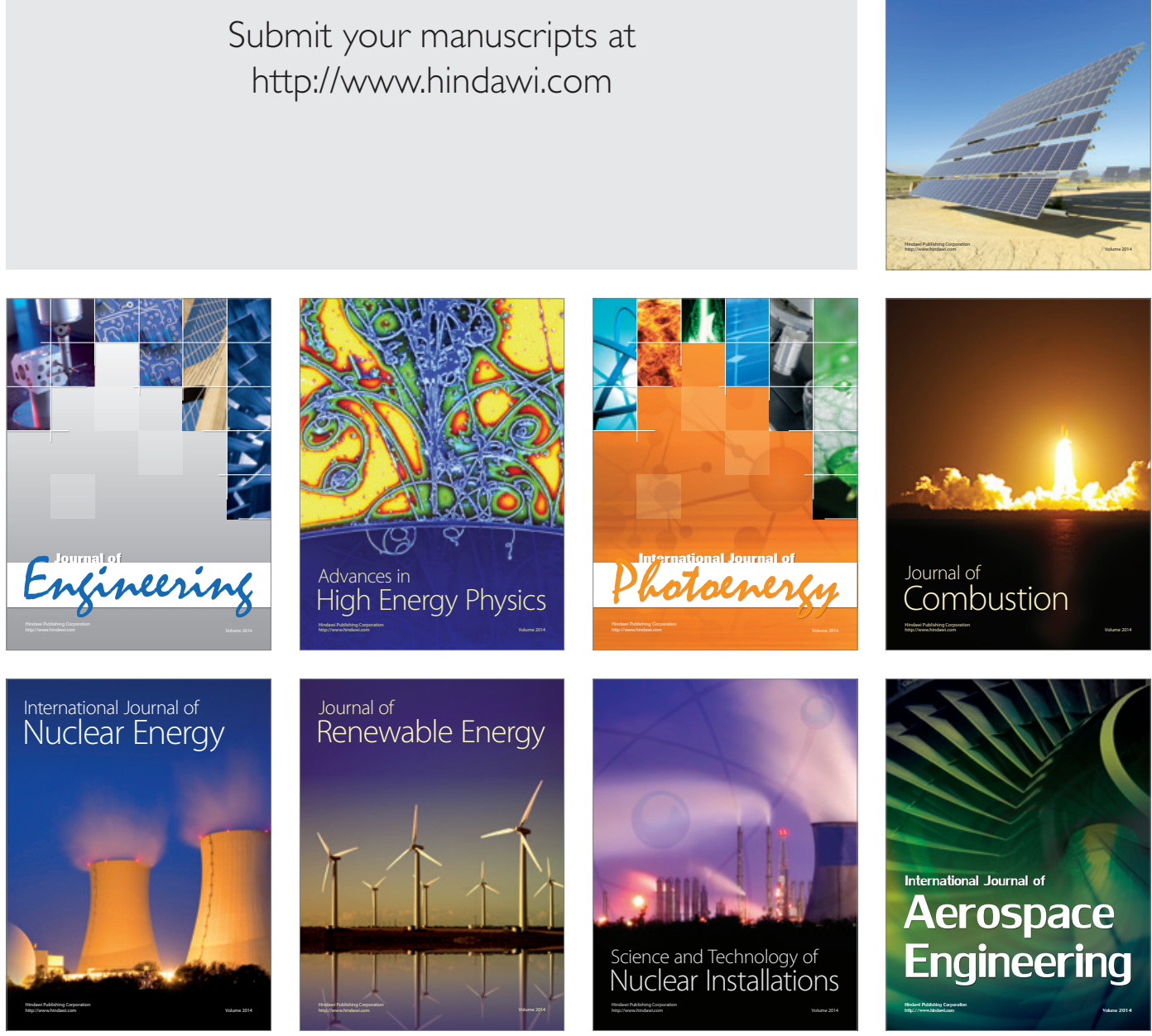